\title{
Cancer Stem Cell of Digestive Organs
}

\author{
Masaki Mori \\ Department of Surgery, Osaka University
}

Recent studies supported the notion that a small population, which mimics norm al adult stem cells in the dormant phase of the cell cycle, plays a role in th e biological behaviors of tumors. Indeed such distinct cells, i.e., cancer ste m cells are resistant to toxic injuries and chemoradiation therapy in vitro an d in vivo. After possible involvement was indicated in leukemia, we were able to report cancer stem cells in gastrointestinal tumors. Our exploration of new screening for surface markers were supposed to be beneficial to identify gast rointestinal cancer stem cells, followed by characterization of chemoresistanc e and tumorigenicity, indicating that several cell surface markers including CD13/APN play a role in biological function of cancer stem cells. Furthermore, we examined the possible effects of cellular reprogramming by induction or inh ibition of cancer-related genes and immature status-related genes including th at of induced pluripotent stem (iPS) cell genes, whose alterations have been $r$ eported in gastrointestinal cancer cells. Introduction of iPS cell genes but a lso several microRNAs, including miR302 was necessary for inducing the express ion of immature status-related proteins and the possible expression of morphol ogical patterns and showed slow proliferation and were sensitized to different iation-inducing treatment, and in vivo tumorigenesis was reduced in nonobese d iabetic mice with severe combined immunodeficiency. Taken together the present study indicates that the combination of traditional therapies with targeted c ancer stem cell-specific agents may target the whole tumors and may offer a pr omising strategy for lasting treatment and even cure. 